\title{
APLICABILIDADE DA CLASSIFICAÇÃO DE ROBSON NA AVALIAÇÃO DAS CESARIANAS NA MATERNIDADE ESCOLA DE VALENÇA - RJ
}

\author{
Applicability of Robson's classification in the evaluation of cesarean sections at \\ maternity school of Valença - RJ
}

Marina Coelho de Paulo ${ }^{1}$ (D) João Alfredo Seixas ${ }^{1}$ Filomena Aste Silveira ${ }^{1}$ (iD) Isadora Faria Silva ${ }^{1}$ Philippe Godefroy ${ }^{1}$

${ }^{1}$ Centro Universitário de Valença (UNIFAA) Valença $(R J)$

\section{Autor correspondente:}

João Alfredo Seixas

E-mail: jalseixas@gmail.com

\section{Como citar este artigo:}

PAULO, M. C. et al. Aplicabilidade da classificação de Robson na avaliação das cesarianas na Maternidade Escola de Valença - RJ. Revista Saber Digital, v. 14, n. 2, p. 56-68, 2021.

Data de submissão: 29/03/2021

Data de aprovação: 10/07/2021 Data de publicação: 21/08/2021

\section{(c) (1) (8)}

Esta obra está licenciada com uma licença http://creativecommons.org/licenses/by-nc/4.0/

\section{RESUMO}

Introdução: O parto cesáreo é indicado em situações em que não é possível a realização do parto vaginal, representando $52 \%$ dos partos na rede pública. Objetivo do estudo: coletar e analisar dados referentes aos partos cesáreos realizados na nossa maternidade, a fim de reduzir sua incidência. Material e Métodos: Trata-se de um estudo descritivo e observacional, em análise de prontuário, onde as gestantes foram classificadas de acordo com o Sistema de Classificação de Robson em dez grupos, onde, em cada grupo, são classificadas de acordo com suas características, que são: paridade (nulípara, multípara com e sem cesariana prévia); início de parto (cesariana espontânea, induzida ou pré-parto), idade gestacional (pré-termo ou termo), apresentação fetal (cefálica, córmica ou transversal) e número de fetos (um ou mais de um). Resultados: Foram analisados 727 prontuários da Maternidade Escola de Valença- RJ, no período de Julho de 2018 a Julho de 2020. No estudo, concluímos que o grupo 5 é o de maior representação (40\%), demonstrando que é comum a realização de cesarianas em pacientes com cesárea anterior, e o grupo 2 representa a segunda maior contribuição, (22\%), evidenciando que devem ser implementadas medidas para reduzir a taxa de realização de cesarianas em nulíparas. Conclusão: Medidas devem ser implementadas na Maternidade Escola de Valença para diminuir a incidência de cesáreas, através da capacitação dos profissionais, e para isso, seria positivo a utilização da Classificação de Robson na avaliação das gestantes e implementação de estratégias de informação e esclarecimento das gestantes

Palavras-chave: Cesárea, Saúde materna, Taxa de gravidez.

\section{ABSTRACT}

Introduction: Cesarean delivery is indicated in situations where vaginal delivery is not possible, representing $52 \%$ of the deliveries in the public health network. Objective: to collect and analyze data referring to caesarean deliveries performed at our maternity, in order to reduce its rates. Materials and Methods: It is a descriptive and observational study, based on medical records analysis, in which the pregnant women were classified according to the Robson Classification System in ten groups, where, in each group, they are classified according to their characteristics, which are: parity (nulliparous, multiparous with and without previous cesarean section); onset of delivery (spontaneous, induced or pre-delivery cesarean section), gestational age (preterm or term), fetal presentation (cephalic, chromic or transverse); and number of fetuses (one or more than one). Results: 728 medical records the Maternidade Escola de Valença - RJ, from July 2018 to July 2020, were analyzed. In the study, we concluded that group 5 was the most representative (40\%), showing that is cesarean deliveries are commonly performed in patients that performed a previous cesarean delivery, and group 2 representes the second bigest contribuition $(22 \%)$, showing that mesures must me implemented to reduce the rate of cesarian deliveries performed in nulliparous. Conclusion: Measures must be implemented at the Maternidade Escola de Valença to reduce the incidence of cesarean, through the training of professionals - in which the use of Robson Classification in the 
evaluation of pregnant women and the implementation of strategies to inform and enlight pregnant women would be positive.

Keywords: Caesarean section; Maternal health; Pregnancy rates.

\section{INTRODUÇÃO}

Nos primórdios da civilização, a cesariana era um procedimento reservado às gestantes que morriam no parto, para salvar o bebê ou para que este pudesse ser sepultado separadamente da mãe. A realização da cesariana na prática obstétrica, só teve início a partir do século XVIII, porém havia alta mortalidade fetal e materna, só sendo praticada em casos muito especiais e somente no século XX a cesárea tornou-se uma operação rotineira (REZENDE, 2009).

As razões específicas para o aumento das taxas de cesáreas na maior parte do mundo permanecem incertas, porém esse aumento é uma grande preocupação de saúde pública e causa um debate considerável devido aos riscos maternos e perinatais, assim como as questões de custo e iniquidade ao acesso. Para propor e implementar medidas eficazes para reduzir as taxas de cesáreas, é necessário identificar quais grupos de mulheres estão sendo mais submetidas ao procedimento e em seguida, investigar suas indicações (TAPIA; BETRAN; GONZALES, 2016).

Desde 1985, a Organização Mundial de Saúde (OMS) considera que a taxa ideal de cesárea fique entre 10\% e 15\%. Segundo a OMS, em 2016, o Brasil deteve a segunda maior taxa de cesáreas do planeta com $55 \%$, perdendo apenas para a República Dominicana, onde a taxa é de 56\%. Esses dados não diferem muito da nossa realidade, onde a taxa de cesárea da nossa maternidade se encontra em $50 \%$. Para uma melhor comparação, a taxa de cesáreas na Europa é de $25 \%$, e nos EUA, 32,8\%. A OMS alerta que a situação já é vista como uma "epidemia". Tanto a OMS como a Federação Internacional de Ginecologia e Obstetrícia (FIGO) recomendam a classificação de Robson como um padrão global para avaliar, monitorar e comparar as taxas de cesáreas dentro 
de um ambiente diversificado com os cuidados da saúde ao longo do tempo e entre instalações (REZENDE, 2009).

A Classificação de Robson tem como objetivo monitorar as taxas de cesárea, ao realizar comparações em padrões já estabelecidos e identificar quais são os grupos específicos que estão mais presentes para a taxa geral, o que torna também a classificação uma ferramenta para ciclos de auditoria, feedback e melhora na assistência (BOATIN et al., 2018).

O sistema de classificação de Robson utiliza características obstétricas básicas para categorizar todas as mulheres admitidas, com sua colocação em um dos dez grupos mutuamente exclusivos e totalmente incluídos. Os principais pontos fortes da Classificação de Robson são a simplicidade de seu design, a validade de sua finalidade, sua facilidade de utilização e a franqueza na interpretação inicial(BETRÁN et al., 2014; BOATIN et al., 2018).

Nossa pesquisa teve como objetivo coletar informações referentes a partos cesariana na nossa maternidade, coma finalidade de contribuir com dados para a gestão da Maternidade Escola, sendo uma possível base para planejamento operativo e estratégico para as equipes, fornecendo melhorias na saúde das nossas gestantes (CANANÉA, [s.d.]). Ademais, é um estudo justificado pela necessidade de medidas de redução da indicação de cesarianas no município de Valença - RJ, em situações em que não deveriam ser indicadas.

\section{MATERIAIS E MÉTODOS}

Trata-se de um estudo descritivo e observacional, realizado no setor de arquivos da Maternidade Escola de Valença, no período de julho de 2018 a julho de 2020, analisando as principais indicações, de acordo com os critérios da Classificação de Robson. Foram coletados dados em prontuários referentes às gestantes que tiveram parto cesáreo na maternidade nesse período, sendo analisados um total de 727 prontuários. 
As gestantes foram classificadas de acordo com os 10 grupos presentes na Classificação de Robson, que divide as gestantes de acordo com cinco características obstétricas, que são: paridade (nulípara, multípara com e sem cesariana prévia); início de parto (cesariana espontânea, induzida ou préparto), idade gestacional (pré-termo ou termo), apresentação fetal (cefálica, córmica ou transversal) e número de fetos (um ou mais de um). Como descrito na tabela 1

\section{Tabela 1 - Classificação de Robson}

\begin{tabular}{|c|c|}
\hline Grupos & \\
\hline 1 & $\begin{array}{l}\text { Nulíparas com feto único, cefálico, maior ou igual a } 37 \text { semanas, } \\
\text { com trabalho de parto espontâneo. }\end{array}$ \\
\hline 2 & $\begin{array}{l}\text { Nulíparas com feto único, cefálico, maior ou igual a } 37 \text { semanas, } \\
\text { cujo parto é induzido ou são submetidas a cesariana antes do início } \\
\text { do trabalho de parto. }\end{array}$ \\
\hline 3 & $\begin{array}{l}\text { Multíparas sem cesárea anterior, com feto único, cefálico, maior ou } \\
\text { igual a } 37 \text { semanas, com trabalho de parto espontâneo. }\end{array}$ \\
\hline 4 & $\begin{array}{l}\text { Multíparas sem cesárea anterior, com feto único, cefálico, maior ou } \\
\text { igual a } 37 \text { semanas, cujo parto é induzido ou que são submetidas à } \\
\text { cesárea antes do início do trabalho de parto. }\end{array}$ \\
\hline 5 & $\begin{array}{l}\text { Todas as multíparas com pelo menos uma cesárea anterior, com } \\
\text { feto único, cefálico, maior ou igual a } 37 \text { semanas. }\end{array}$ \\
\hline 6 & Todas as nulíparas com feto único em apresentação pélvica. \\
\hline 7 & $\begin{array}{l}\text { Todas multíparas com feto único em apresentação pélvica, incluindo } \\
\text { aquelas com cesariana anterior (es). }\end{array}$ \\
\hline 8 & $\begin{array}{l}\text { Todas as mulheres com gestação múltipla, incluindo aquelas com } \\
\text { cesariana (s) anterior (es). }\end{array}$ \\
\hline 9 & $\begin{array}{l}\text { Todas as gestantes com feto em situação transversa ou oblíqua, } \\
\text { incluindo aquelas com cesárea (s) anterior (es). }\end{array}$ \\
\hline
\end{tabular}




\begin{tabular}{|l|l|}
\hline 10 & $\begin{array}{l}\text { Todas as gestantes com feto único e cefálico, menor que } 37 \\
\text { semanas, incluindo aquelas com cesárea (s) anterior (es). }\end{array}$ \\
\hline
\end{tabular}

Os dados foram consolidados no programa Excel. As gestantes foram classificadas de acordo com os grupos, durante o período estimado para essa pesquisa e a contribuição de cada grupo foi avaliada para propor mudanças na taxa total de cesáreas.

\section{RESULTADOS}

Do total dos 727prontuários analisados, 15 foram excluídos, por não possuírem anotações que possibilitassem a sua classificação. Foram Totalizados então 712 prontuários.

A contribuição de cada grupo, de acordo com a Classificação de Robson, em relação ás cesáreas realizadas no período avaliado, está descrita na Figura 1.

Figura 1- Percentual de cesáreas levando em consideração a Classificação de Robson.

\section{Porcentagem}

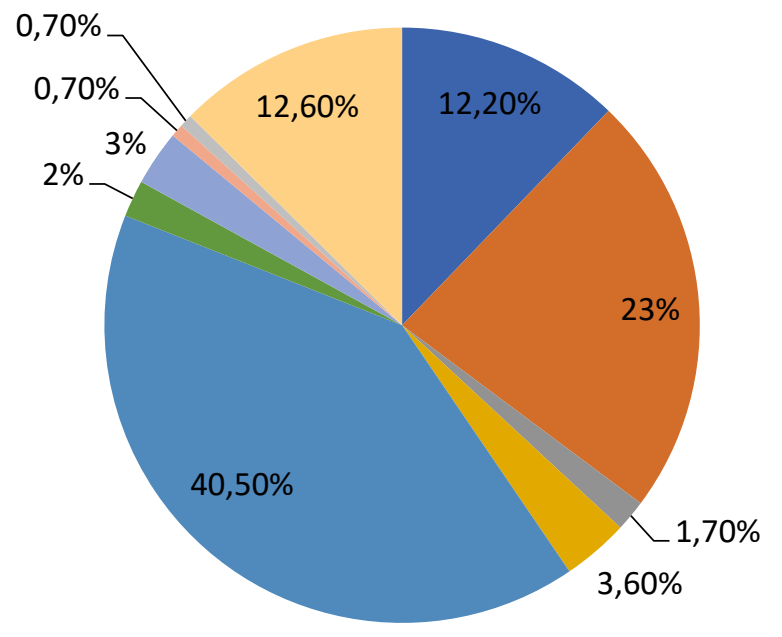

Grupo 1

Grupo 2

Grupo 3

Grupo 4

Grupo 5

Grupo 6

Grupo 7

Grupo 8

Grupo 9

Grupo 10 
A caracterização de cada grupo, de acordo com a Classificação Mundial de Robson, está representada na figura 2, de acordo com a quantidade em cada grupo.

Figura 2 - Quantidade de cesáreas distribuídas em cada grupo.

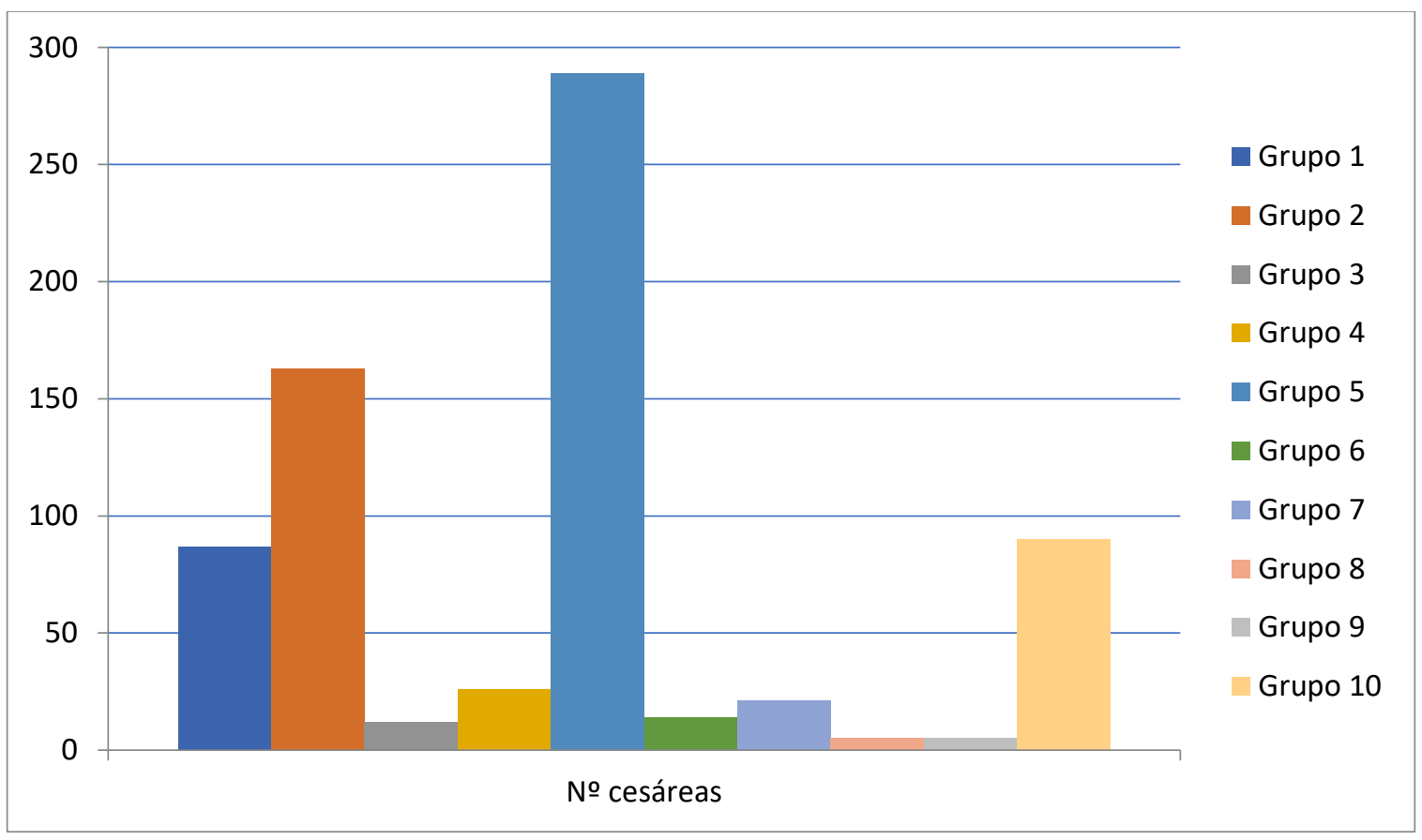

\section{DISCUSSÃO}

Para auxiliar os parâmetros que orientam a realização da cesárea, a OMS, em 10 de abril de 2015, propôs utilização da Classificação de Robson como padrão global, considerando taxas ideais das cesáreas entre 10 a 15\%. Quando as taxas de cesáreas são maiores do que $10 \%$ a nível populacional, essas taxas não estão associadas a redução da mortalidade materna e neonatal. As consequências negativas desse número elevado de cesáreas, estão presentes principalmente em mulheres com baixo acesso a serviços e cuidados obstétricos, seja pela infraestrutura, tratamento de complicações pós operatórias como infecções ou cirurgias realizadas de forma não segura (WHO, 2001). 
No presente estudo, o grupo 5 representou a grande parcela de cesáreas no período analisado. Isso vem de encontro com os números encontrados a nível nacional, no Brasil, o grupo que mais impactou no número de cesáreas, foram as multíparas com parto cesáreo anterior (NAKAMURA-PEREIRA et al., 2016). Ao analisarmos quais seriam os motivos para essa estatística, podemos citar que diversos médicos temem as complicações decorrentes de um parto vaginal após uma cesárea anterior e como exemplo, podemos citar a rotura uterina. Estudos observacionais descrevem que mesmo após uma cesárea, é possível que um parto vaginal seja realizado com segurança, demonstrando que o sucesso de um parto vaginal após uma cesárea prévia, tem risco de rotura uterina inferior a 1\% (FLAMM et al., 1994).

Outro fato interessante é que grande parcela das gestantes incluídas no grupo 5, é abordada dessa forma pelo motivo da realização de uma laqueadura tubária. No Brasil, principalmente na rede pública, a dificuldade de acesso ao planejamento familiar pode ser um fator que induz a realização de cesáreas, visto que a cirurgia de laqueadura, se corretamente indicada, poderá ser realizada naquela mesma cirurgia. Em situações em que as gestantes manifestem sua preferência pela cesariana, é dever do profissional de saúde avaliar os riscos e benefícios, e razões específicas para tal procedimento. Em alguns casos, por exemplo, estratégias de apoio psicológico e orientações sobre os métodos para alívio da dor do parto vaginal são extremamente importantes (BARBOSA et al., 2003).

Recentemente, uma análise da OMS descobriu que a taxa de cesáreas e a contribuição absoluta do grupo 5 aumentaram nos últimos anos. Esses dados mostram o efeito dominó do uso de cesáreas, com taxas crescentes, especialmente em mulheres nulíparas, aumentam o número de mulheres com cesárea anterior, com maior probabilidade de sofrer uma repetição do procedimento (NAKAMURA-PEREIRA et al., 2016). 
Nossos achados estão de acordo com os achados de MENDES (2021), que mostrou que a proporção de mulheres do Grupo 5, foi o dobro do parâmetro da OMS (MENDES; RATTNER, 2021).

Dando sequência, o grupo de Robson que teve a segunda maior porcentagem foi o grupo 2, em que estão incluídas nulíparas com feto único, cefálico, a termo, cujo parto é induzido ou que foram submetidas a cesárea antes do início do trabalho de parto. É importante para estas gestantes nulíparas, que seja realizada orientação em relação ao trabalho de parto, e sejam utilizadas medidas não farmacológicas para uma melhor experiência, como por exemplo, banhos, técnicas de respiração, acupuntura, aromaterapia e utilização da bola suíça. (SANTOS et al., 2021) Um excelente parâmetro que deve ser utilizado pelo serviço é o partograma, que nos permite acompanhar a evolução e diagnosticar intercorrências de forma precoce durante o trabalho de parto.É importante salientar que os grupos 1 e 2, tem grande chance de evoluir para o grupo 5 na gestação a seguir, porque, consequentemente, suas posteriores gestações também tendem a cesárea (CECATTI et al., 2000; HADDAD, 2011).

Em outro estudo, o maior grupo isolado foi o grupo 2, compreendendo 20\% de toda a população (NAKAMURA-PEREIRA et al., 2016).

Estudos relatam que em grande parte das vezes, a falha na progressão do trabalho de parto é consequência de contrações uterinas deficientes, e desproporções céfalo-pélvicas. Por isso, a primeira intervenção é estimular essas contrações, seja com administração de ocitocina, ou com a ruptura das membranas. Ademais, se o parto não ocorrer com essas medidas, devem ser considerados fatores mecânicos, como a desproporção céfalo pélvica, por exemplo. Portanto, as nulíparas que não foram admitidas em trabalho de parto e que após estimulação da contração uterina não evoluem no trabalho de parto, serão, indiscutivelmente, submetidas à cesariana (CECATTI et al., 2000).

Os grupos 1 e 10 apresentaram a mesma proporção, com aproximadamente $12 \%$ de representação. Estão incluídas no grupo 1 as nulíparas com feto único, cefálico, a termo e em trabalho de parto espontâneo, 
e no grupo 10 todas as gestantes com feto único, cefálico, porém com idade gestacional menor que 37 semanas. Dentre os principais motivos para indicação de cesariana em nulíparas do grupo 1, podemos destacar os fatores mecânicos, como a desproporção céfalo pélvica e fatores relacionados a saúde do feto, como frequência cardíaca não tranquilizadora, centralização, sofrimento fetal agudo(AMORIM, 2010; SOUZA, 2010). O grupo 10 também tem grande parcela de representação o que evidencia que o serviço da Maternidade Escola de Valença, referência na região sul Fluminense, têm investido em tecnologia e capacitação da equipe multidisciplinar na questão da assistência ao parto prematuro.

Outro estudo identificou a contribuição dos grupos 5, 1 e 2, para mais de $50 \%$ das cesarianas realizadas em várias instituições de diferentes países e continentes. A ocorrência de cesariana nos 10 grupos de Robson também foi alta, sendo o grupo 3 a única exceção(BOLOGNANI et al., 2018).

No estudo de NAKAMURA (2016), o grupo único, cefálico, pré-termo (grupo 10) representou quase $10 \%$ dos nascimentos(NAKAMURA-PEREIRA et al., 2016).

Podemos realizar uma comparação do grupo 5, o mais numeroso, com os outros grupos também compostos por multíparas, porém sem cesárea anterior. Os grupos 3 e 4, que tiveram, respectivamente $2 \%$ e $4 \%$ no presente estudo, ambos incluem multíparas, sem cesárea anterior, com feto a termo, e diferem entre si em relação ao trabalho de parto, que no grupo 3 é de forma espontânea, e no grupo 4 foi necessário indução ou cesárea sem início do trabalho de parto. Especialmente em multíparas sem cesárea anterior, inúmeras indicações estão presentes e podemos citar, por exemplo, descolamento prematuro de placenta, rotura uterina, placenta prévia, pré-eclâmpsia grave e eclampsia, infecção pelo vírus da imunodeficiência humana (HIV), cardiopatia materna, malformação fetal, sofrimento fetal crônico, prematuridade, desproporção céfalo-pélvica, prolapso, procidência e circular de cordão umbilical e sofrimento fetal agudo(CECATTI et al., 2000; HADDAD, 2011). 
Os grupos de multíparas sem cesárea (grupos 3 e 4) contribuíram para pouco mais de 10\% das cesáreas no estudo de NAKAMURA-PEREIRA (2016).

As taxas de cesáreas aumentaram com o tempo devido ao aumento do procedimento em grupos com trabalho de parto espontâneo (grupos 1 e 3) e no grupo de multíparas com cesárea anterior e com uma gravidez cefálica a termo, visto em outros estudos (TAPIA; BETRAN; GONZALES, 2016).

A maior contribuição para a taxa global de cesárea foi observada nos grupos 1, 2 e 5 . Esses grupos foram identificados como "grupos-alvo" por serem responsáveis por $67,5 \%$ de todos as cesáreas do estudo, sendo o grupo 50 maior contribuinte (KACERAUSKIENE et al., 2017).

O grupo 8 tem como composição as gestações múltiplas e no presente estudo teve menos que $1 \%$ de representação. A via de parto das gestações múltiplas apresenta controvérsias. A recomendação mais aceita é de que em gestações gemelares não complicadas, com primeiro gemelar em apresentação cefálica, devemos realizar a tentativa de um parto vaginal. Nesse caso, a cesariana eletiva não mudará o fato de que a morbidade e mortalidade perinatal do segundo feto é aumentada e não deve ser indicada. Entretanto, quando o primeiro feto não está cefálico, grande parte das literaturas indica cesariana. É muito importante salientar que independente da apresentação, as cesáreas em gemelares somente devem ser indicadas a partir da 38ª semana da gravidez e devem ser realizadas antes somente se situação fetal não tranquilizadora (AMORIM, 2010; SOUZA, 2010).

Em última análise, temos os grupos 6, 7 e 9. No presente estudo, eles representam respectivamente $2 \%, 3 \%$ e $0,7 \%$. O grupo 6 inclui todas as nulíparas com feto único, em apresentação pélvica, e o grupo 7 as multíparas em apresentação pélvica. Uma revisão sistemática constatou que nessa situação, deve-se considerar o desejo da mãe e principalmente a segurança e habilidade do obstetra para conduzir o parto pélvico. Além disso, a gestante deve ser sempre informada dos riscos perinatal e neonatal, através de um termo de consentimento (HOFMEYR; HANNAH, 2003). 
No estudo de NAKAMURA (2016), os grupos de apresentações não cefálicas (grupos 6, 7 e 9) e gêmeos (grupo 8) juntos, contribuíram com apenas 8,9\% das cesarianas (NAKAMURA-PEREIRA et al., 2016).

No grupo 9, que teve uma parcela de $2 \%$, estão incluídas todas as gestantes em situação transversa ou oblíqua, incluindo aquelas com cesárea anterior. É uma indicação absoluta de parto cesáreo e podem ser necessárias investigações complementares dessa apresentação anômala ainda no pré-natal (AMORIM, 2010; SOUZA, 2010).

Em consonância a todos esses fatores acima descritos, devemos considerar também que a maior parte da população brasileira é atendida pelo SUS e na maioria das vezes, o médico que acompanha o pré-natal dificilmente será o profissional que irá realizar o parto. Isso enfatiza a necessidade de um serviço acolhedor para que não haja uma desvinculação entre o pré-natal e o parto propriamente dito. Acrescenta-se a realização de cesáreas sem indicação pela ausência de informações de toda a gestação em curso e até mesmo dos antecedentes obstétricos(HOTIMSKY et al., 2002).

\section{CONCLUSÃO}

É possível concluir que a classificação de Robson de acordo com os 10 grupos deve ser adotada na Maternidade Escola de Valença com o objetivo de identificar e posteriormente buscar e aplicar soluções para reduzir as taxas de cesáreas de forma alinhada com o padrão da OMS.

O Sistema de classificação de Robson é um modelo prático e a equipe multidisciplinar do hospital escola deve ser capacitada a adotá-la. Essa classificação permite que seja realizada uma análise temporal dos dados a fim de identificar quais são os grupos que representam a maior parcela, comparando-os com os dados fornecidos pela OMS. 
Mostramos em nosso estudo que a classificação de Robson pode funcionar não apenas para identificar os grupos-alvo de mulheres que mais influenciam a taxa de cesarianas, mas pode funcionar como uma ferramenta de auditoria para identificar os grupos que podem se beneficiar de estratégias e intervenções, focadas na redução das taxas de cesariana na Maternidade do Hospital Escola de Valença.

\section{REFERÊNCIAS}

AMORIM, M. Indicações de cesariana baseadas em evidências: parte I. FEMINA, v. 38, n. 8, p. 459-468, 2010.

BARBOSA, G. P. et al. Parto cesáreo: quem o deseja? Em quais circunstâncias? Cadernos de Saúde Pública, v. 19, n. 6, p. 1611-1620, 2003.

BETRÁN, A. P. et al. A Systematic Review of the Robson Classification for Caesarean Section: What Works, Doesn't Work and How to Improve It. PLoS ONE, v. 9, n. 6, p. e97769, 2014.

BOATIN, A. et al. Audit and feedback using the Robson classification to reduce caesarean section rates: a systematic review. BJOG: An International Journal of Obstetrics \& Gynaecology, v. 125, n. 1, p. 36-42, 2018.

BOLOGNANI, C. V. et al. Robson 10-groups classification system to access Csection in two public hospitals of the Federal District/Brazil. PLOS ONE, v. 13, n. 2, p. e0192997, 2018.

CANANÉA, B. Use of the Robson Classification in reducing the rate of cesarean section. Brazilian Journal of Development Braz, v. 6, n. 11, p. 89043-89053, 2020.

CECATTI, J. G. et al. Fatores Associados à Realização de Cesárea em Primíparas com uma Cesárea Anterior. Revista Brasileira de Ginecologia e Obstetrícia, v. 22, n. 3, 2000.

FLAMM, B. L. et al. Elective Repeat Cesarean Delivery Versus Trial of Labor: A Prospective Multicenter Study. Obstetrics \& Gynecology, v. 83, n. 6, p. 927932, 1994. 
HADDAD, S. Estratégias dirigidas aos profissionais para a redução das cesáreas desnecessárias no Brasil. Revista Brasileira de Ginecologia e Obstetrícia, v. 33, n. 5, p. 252-262, 2011.

HOFMEYR, G. J.; HANNAH, M. E. Planned caesarean section for term breech delivery. The Cochrane Database of Systematic Reviews, n. 3, p. CD000166, 2003.

HOTIMSKY, S. N. et al. O parto como eu vejo... ou como eu o desejo?: expectativas de gestantes, usuárias do SUS, acerca do parto e da assistência obstétrica. Cadernos de Saúde Pública, v. 18, n. 5, p. 1303-1311, 2002.

KACERAUSKIENE, J. et al. Implementation of the Robson classification in clinical practice:Lithuania's experience. BMC Pregnancy and Childbirth, v. 17, n. 1, p. 432, 2017.

MENDES, Y. M. M. B. E; RATTNER, D. Cesarean sections in Brazil's teaching hospitals: an analysis using Robson Classification. Revista Panamericana de Salud Pública, v. 45, p. 1, 2021.

NAKAMURA-PEREIRA, $M$. et al. Use of Robson classification to assess cesarean section rate in Brazil: the role of source of payment for childbirth. Reproductive Health, v. 13, n. S3, p. 128, 2016.

REZENDE, J. M. DE. À sombra do Plátano: crônicas de história da medicina. São Paulo: Editora Fap-Unifesp, 2009.

SANTOS, A. C. DE M. et al. ATUAÇÃO DA ENFERMAGEM NO USO DE MÉTODOS NÃO FARMACOLÓGICOS PARA O ALÍVIO DA DOR DURANTE O TRABALHO DE PARTO/ URSING PERFORMANCE IN THE USE OF NONPHARMACOLOGICAL METHODS FOR PAIN RELIEF DURING CHILD LABOR. Brazilian Journal of Development, v. 7, n. 1, p. 9505-9515, 2021.

SOUZA, A. Indicações de cesariana baseadas em evidências: parte II. FEMINA, v. 38, n. 9, p. 459-468, 2010.

TAPIA, V.; BETRAN, A. P.; GONZALES, G. F. Caesarean Section in Peru: Analysis of Trends Using the Robson Classification System. PLOS ONE, v. 11, n. 2, p. e0148138, 2016.

WHO. WHO Statement on Caesarean Section Rates, 2001. 